Canadian

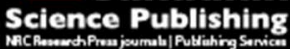

Applied Physiology, Nutrition, and Metabolism Physiologie appliquée, nutrition et métabolisme

\title{
Validity of field assessments to predict peak muscle power in preschoolers
}

\begin{tabular}{|r|l|}
\hline Journal: & Applied Physiology, Nutrition, and Metabolism \\
\hline Manuscript ID & apnm-2016-0426.R2 \\
\hline Manuscript Type: & Article \\
\hline Date Submitted by the Author: & $16-$ Mar-2017 \\
\hline Complete List of Authors: & $\begin{array}{l}\text { King-Dowling, Sara; McMaster University } \\
\text { Proudfoot, Nicole; McMaster University, Pediatrics } \\
\text { Cairney, John; McMaster University, Family Medicine/Kinesiology } \\
\text { Timmons, Brian; McMaster University, }\end{array}$ \\
\hline Keyword: & $\begin{array}{l}\text { children, standing long jump, standing broad jump, musculoskeletal } \\
\text { fitness, shuttle run }\end{array}$ \\
\hline &
\end{tabular}


Validity of field assessments to predict peak muscle power in preschoolers

\author{
Sara King-Dowling, PhD Candidate ${ }^{1,2,3}$ \\ Nicole A. Proudfoot, MSc, PhD Candidate ${ }^{1,2}$ \\ John Cairney, $\mathrm{PhD}^{1,3}$ \\ Brian W. Timmons, $\mathrm{PhD}^{1,2,3}$
}

${ }^{1}$ Department of Kinesiology, McMaster University, Hamilton, Canada

${ }^{2}$ Child Health and Exercise Medicine Program, Department of Pediatrics, McMaster University, Hamilton, Canada

${ }^{3}$ Infant and Child Health Lab, Department of Family Medicine, McMaster University, Hamilton, Canada

*Corresponding author: Brian W. Timmons, PhD, Child Health \& Exercise Medicine Program Department of Pediatrics, McMaster University; Address: 1280 Main Street West, HSC 3N27G Hamilton, ON, Canada, L8S 4K1; Tel: 905-521-2100 ext 77615; Fax: 905-521-1703; Email: timmonbw@,mcmaster.ca 


\begin{abstract}
Background: Field-based fitness assessments are time- and cost-efficient. However, no studies to date have reported the predictive value of field-based musculoskeletal fitness assessments in preschoolers. The purpose of this study was to determine the validity of two field assessments to predict peak muscle power in preschool-aged children.
\end{abstract}

Methods: Four-hundred nineteen 3- to 5-year olds participated (208 girls, 211 boys; mean age: $4.5 \pm 0.9$ years). Peak power (PP) was evaluated using a modified 10 -second Wingate protocol as the criterion standard. Standing long jump was measured in inches to the back of the heel using a 2-footed takeoff and landing. Shuttle run time was measured using a shuttle run protocol, that required children to sprint 50 feet $(15.2 \mathrm{~m})$, pick up a small block and sprint back, with time measured to the closest millisecond. Regression modeling was used to calculate the predictive power of each field-based measurement, adjusting for weight $(\mathrm{kg})$, age and sex.

Results: Both standing long jump distance and shuttle run time were significantly correlated with PP $(\mathrm{r}=0.636, \mathrm{p}<.001$ and $\mathrm{r}=-0.684, \mathrm{p}<.001$ respectively $)$. Linear regression analysis determined that a child's PP can be predicted from the child's weight, age and sex and either standing long jump or shuttle run time (adjusted $\mathrm{R}^{2}=0.79, \mathrm{p}<.001$ and $0.81, \mathrm{p}<.001$, respectively).

Conclusion: The standing long jump and the BOT-2 shuttle run are both significant predictors of peak muscle power in preschool children. Either measure can be used as a cost and time efficient estimate of musculoskeletal fitness in preschoolers.

Key Words: Children; standing long jump; standing broad jump; musculoskeletal fitness; shuttle run, short-term muscle power 


\section{INTRODUCTION}

Musculoskeletal fitness (MF) is now recognized as an important marker of health across the life span (Pate et al. 2012). Emerging evidence shows positive associations between MF and bone heath and self-esteem as well as negative associations between MF and central adiposity, cardiovascular disease and metabolic risk factors among children and adolescents (Ortega et al. 2008, Smith et al. 2014). Long-term follow-up studies have also found that, compared to cardiorespiratory fitness, measures of MF in adolescence are more strongly predictive of bone mineral density later in life (Kemper et al. 2000, Barnekow-Bergkvist et al. 2006).

Despite the growing body of work on MF in childhood, a recent review by Plowman and colleagues (2014) concluded that many important questions still remain regarding how we test MF in children and youth. As population level studies rely heavily on field-based assessment of fitness, the first step to understanding the impact of MF on health in childhood is to determine how well MF field-tests agree with criterion laboratory measures of the same construct. The use of valid and reliable MF field tests will make it possible to measure meaningful changes and deficits over time and relate this back to important health outcomes in large samples of children.

One important aspect of MF is muscle power (Pate et al. 2012). Short-term muscle power, or peak power is defined as the highest mechanical power that can be delivered during short duration exercise $(\leq 30 \mathrm{~s})$, with 'true' peak power requiring measures of instantaneous force and velocity (Van Praagh and Doré 2002). The Wingate anaerobic test is a 30 second cycle ergometer sprint test used to measure peak power, mean power and percent fatigue (Bar-Or 1987). It is one of the most common methods for assessing short-term muscle power; however, 
the specialized equipment is costly and not feasible in large population -based studies, especially in school/preschool settings.

Although there is growing evidence supporting MF field tests in school-aged children (Safrit 1990, Freedson et al. 2000, Castro-Piñero et al. 2010a, Artero et al. 2011), much less is known about the validity of MF testing in the kindergarten/preschool population. As young children tend to accumulate their daily physical activity in short, intense intermittent bouts (Baquet et al. 2007), short-term muscle power may be extremely relevant to their habitual activity patterns. In a recent systematic review by Ortega and colleagues (Ortega et al. 2015), the authors identified the need for validation studies as no studies to date have examined the criterion-related validity of field-based MF assessments in preschoolers. The standing long jump (standing broad jump) and Bruininks Oseretsky Test of Motor Proficiency $2^{\text {nd }}$ Edition (BOT-2, (Bruininks and Bruininks 2005)) shuttle run were examined as they are field-based tests that are easy to administer and comprehend, time efficient, and involve minimal equipment, making them ideal for field-based fitness testing in a young population. The purpose of this study was to determine if the standing long jump (LJ) and the BOT-2 shuttle run are able to accurately predict peak muscle power, as measured using a modified 10s Wingate protocol as the criterion standard (Nguyen et al. 2011), in a community-based sample of preschool children 3-5 years old.

\section{METHODS}

\section{Participants}

Four hundred and nineteen preschool children were recruited from various community organizations in south-central Ontario from 2010-2012 as part of the Health Outcomes and 
Physical activity in Preschoolers (HOPP) Study, which is a longitudinal observational study examining the interrelationships between physical activity and numerous health outcomes from preschool to early childhood. Details of the study design are provided in a previous publication (Timmons et al. 2012). The current study is a cross-sectional secondary analysis of the baseline cohort of children who range in age from 3.0 (36 months) - 5.9 years (71 months) (50.4\% male). Children with known physical impairments or diagnosed medical condition (e.g. diabetes, cerebral palsy) were not eligible to participate. Informed, written consent was given by parents/guardians of all participants. Ethical approval was obtained from the McMaster University Faculty of Health Science/Hamilton Health Sciences Research Ethics Board.

\section{Measures}

Anthropometrics

Standing height was measured without shoes to the nearest $0.1 \mathrm{~cm}$ using a calibrated stadiometer. Weight was measured on a digital scale to the nearest $0.1 \mathrm{~kg}$ in light clothing and without shoes. For descriptive purposes, Body Mass Index (BMI) was calculated as weight $/ \mathrm{height}^{2}\left(\mathrm{~kg} / \mathrm{m}^{2}\right)$. BMI percentiles, based on sex and age, were calculated based on Centers for Disease Control and Prevention (CDC) growth charts (Kuczmarski et al. 2002).

\section{Field Assessments}

\section{Standing Long jump (LJ)}

The standing long jump (LJ) (or standing broad jump) was chosen as is used in numerous fitness test batteries across the world and was a recommended MF field test in the Institute of Medicine's report on youth fitness measurement (Pate et al. 2012). It is considered to be a 
measure of explosive strength/muscle power and has found to be reliable in preschoolers (Oja and Jürimäe 1997, Bénéfice et al. 1999, Artero et al. 2011, Fernandez-Santos et al. 2015) and to be strongly associated with other lower body muscular strength field tests (e.g. vertical jump, counter movement jump, squat jump) in children and youth (Castro-Piñero et al. 2010b, Fernandez-Santos et al. 2015). In addition, the relative simplicity of the long jump compared to other jump protocols such as the counter movement jump and vertical jump, made this test ideal for the preschool population.

Children were required to stand with both feet behind a marked line. Participants were instructed to jump as far as they could off both feet. The jump was first demonstrated using appropriate arm swing and 2 footed takeoff and landing. If the child did not understand the instructions the task was re-demonstrated and any errors were corrected. Distance was measured, to the nearest half inch, from the marked line to the back of the closest heel. Each child performed 3 jumps and the best result was recorded. Jumps were not recorded if the children fell or if they used a one-footed take-off or landing (Ruiz et al. 2011).

\section{BOT-2 shuttle-run}

Speed and agility, although mainly thought to assess 'motor fitness', also involves many components of $\mathrm{MF}$ as it requires explosive lower body strength and power along with balance and coordination (Ortega et al. 2008, Artero et al. 2011).

Running speed and agility was assessed using the Bruininks-Oseretsky Test of Motor Proficiency $2^{\text {nd }}$ Edition (BOT-2, (Bruininks and Bruininks 2005)) shuttle run protocol. This test required 
children to sprint 50 feet $(15.2 \mathrm{~m})$, pick up a small shuttle block and sprint back, which was explained verbally before beginning the test. Children started on a ready-set-go signal from the investigator and were encouraged to run as fast as they could throughout the test. Shuttle run time (SRT) was measured in seconds to the nearest tenth of a second. A second trial was conducted only if the participants stumbled, fell, failed to pick up the shuttle block or dropped the shuttle block before crossing the start/finish line during the first trial. If a second trial was necessary, the task was explained again and then demonstrated by the investigator. The full running speed and agility subsection ( 5 items) of the BOT-2 has been found to have high testretest and inter-rater reliability in young children age 4-7 (Bruininks and Bruininks 2005). Similar shuttle-run tests also have good reliability in preschool children (e.g. $4 \times 10 \mathrm{~m} 4 \times 4 \mathrm{~m}$; Ortega et al. 2015), however this test was chosen in order to limit complexity as only 1 change in direction is required.

Peak muscle power

\section{Modified Wingate Test}

As young children accumulate the majority of their moderate to vigorous physical activity in bouts less than 10 seconds (Baquet et al. 2007), we developed a modified 10s Wingate anaerobic cycling test to determine short-term muscle power (Nguyen et al. 2011). The test was performed on a pediatric cycle ergometer (Fleisch-Metabo, Geneva, Switzerland, or Pediatric Corival, LODE, the Netherlands). The Wingate test is considered a gold standard in short-term muscle power assessment, and this modified 10s protocol has been shown to be feasible and highly reliable (ICC 0.93) for preschoolers 3-5 years old (Nguyen et al. 2011). Seat height and pedal crank length were adjusted for each child and their feet were taped into the pedals. In order to 
establish peak cadence (rpm), the child performed an initial "sprint" by pedaling as fast as they could for $\sim 20 \mathrm{~s}$ against only the internal resistance of the ergometer. After a short rest, the child began the modified Wingate test. The child was instructed to pedal as fast as they could with a "ready... set... go" signal from the investigator. When $80 \%$ of the peak cadence was reached, the braking force $\left(0.55 \mathrm{~N} \cdot \mathrm{m} \cdot \mathrm{kg}^{-1}\right.$ body mass $)$ was applied. During the 10 -second test, the child was encouraged to keep pedalling as fast as he/she could. Peak power (PP) in Watts was taken as the highest instantaneous power output achieved during the test. If children were unable or refused to pedal over $25 \mathrm{rpm}$ they were excluded from the current analysis.

Due to change of equipment from the Fleisch-Metabo to the LODE cycle ergometer after the first 65 participants, a calibration study on 28 participants who completed tests on both ergometers one week apart was conducted (Caldwell et al. 2016). No significant differences in peak or mean power were observed, and therefore results from both ergometers were included in the final analyses.

Statistical Analyses

Sex differences in anthropometrics and fitness measures were examined using independent ttests. Pearson correlations were conducted to examine the relationship between PP and both LJ and SRT. In order to determine the predictive power of each field test, multiple variable linear regression analysis was conducted. Weight $(\mathrm{kg})$, age and sex were first added into the model using the entry method (Model 1). SRT (Model 2) or LJ (Model 3) or both (Model 4) were then entered simultaneously into the model to determine the unique predictive power of each field test over and above weight $(\mathrm{kg})$, age and sex. Age was measured in years and collapsed into three 
age groups (3 (3.0-3.99), 4 (4.0-4.99) and 5 (5.0-5.99) years) in order to make the predictive equations easier and more feasible to use in a field setting. Data analysis was conducted using SPSS v.20.

\section{RESULTS}

\section{Participant Characteristics}

Four hundred and nineteen children (208 girls, 211 boys) participated in the baseline HOPP study assessments. 15 children were not able to pedal $>25 \mathrm{rpm}$ (mean age $3.3 \pm 0.3$ years, 8 boys), 8 children refused (mean age $3.4 \pm 0.4,6$ boys) and 3 children did not have PP values due to illness, unavailable equipment, or equipment malfunction, leaving 393 (94\%) children (198 girls, 195 boys) with valid peak power results. Descriptive characteristics of these children are presented in Table 1. Of the 393 with completed modified 10s Wingate tests, 17 children did not complete the LJ and 13 did not complete the BOT-2 shuttle run (due to refusal/cooperation difficulties $(n=5)$ or time/space restraints during testing sessions). Three of these children did not complete both the LJ and BOT-2 shuttle run and were therefore removed from the field-test analyses entirely. Boys and girls did not differ on $\mathrm{LJ}(\mathrm{t}=1.2, \mathrm{p}=.23)$ or BOT-2 shuttle run ( $\mathrm{t}=1.1$, p=.28) performance; however, boys had significantly higher PP compared to girls (98.7 vs. 89.2 $\mathrm{W} ; \mathrm{t}=2.5, \mathrm{p}=.01)$. All participants had valid height and weight measurements. Boys were significantly taller and heavier $(\mathrm{t}=2.0, \mathrm{p}<.05, \mathrm{t}=2.1, \mathrm{p}<.05$, respectively) and no sex differences in age $(\mathrm{t}=.03, \mathrm{p}=0.98)$ or BMI $(\mathrm{t}=0.9, \mathrm{p}=.35)$ were found. 


\section{Criterion-related Validity}

Both LJ distance and SRT were significantly correlated with PP $(\mathrm{r}=0.636, \mathrm{p}<.001$ and $\mathrm{r}=-0.684$, $\mathrm{p}<.001$, respectively). Linear regression analysis determined that a child's PP (Watts) can be predicted from the child's weight (kg), age (years) and sex and either LJ distance (in) or SRT (s) (adjusted $\mathrm{R}^{2}=0.79, \mathrm{p}<.001$ and $0.81, \mathrm{p}<.001$, respectively). Using both field measures in the same model slightly improved the model's overall accuracy (adjusted $\mathrm{R}^{2}=0.82, \mathrm{p}<.001$ ) (Table 2).

\section{Predictive equations}

Equations that can be used to calculate PP from each of the field tests are below:

LJ

$$
\mathrm{PP}(\text { Watts })=9.0(\text { age })+3.7(\text { sex })+7.1(\text { weight })+0.8(\mathrm{LJ})-97.7
$$

BOT-2 shuttle run

$$
\mathrm{PP}(\text { Watts })=7.3(\text { age })+3.4(\text { sex })+7.3(\text { weight })-5.4(\text { SRT })-4.9
$$

Combined

$\mathrm{PP}($ Watts $)=6.1($ age $)+3.3($ sex $)+6.8($ weight $)+0.5(\mathrm{LJ})-4.3(\mathrm{SRT})-19.1$ age in years $(3,4$ or 5$)$; sex: female $=0$, male $=1$; weight in $\mathrm{kg}$ to the nearest $0.1 \mathrm{~kg}$; LJ: long jump distance to the nearest half inch; SRT: shuttle run time in seconds to the nearest tenth of a second.

\section{DISCUSSION}

Due to the recent emphasis on MF as an important health marker in childhood, it is important to be able to measure this aspect of health using valid assessments outside the laboratory setting. 
Although the Wingate anaerobic test is commonly referred to as a gold standard for the measurement of short-term muscle power, it requires expensive equipment, trained personnel and is not suitable for testing large groups of children. The purpose of this study was to determine the construct validity of the $\mathrm{LJ}$ and $50 \mathrm{ft}$ shuttle run by measuring their ability to accurately predict peak power as measured using a criterion standard modified Wingate protocol in preschool children. The results show that when used in combination with age, weight and sex, both the LJ and BOT-2 shuttle run are able to predict approximately $80 \%$ of the variance in PP in children aged 3-5 years. Although SRT shows slightly better predictive accuracy, either the LJ or SRT can be conducted as cost- and time-efficient estimates of peak muscle power in preschoolers.

Jump tests and sprint times are common MF field measures used in many national fitness protocols (Pate et al. 2012) (e.g. CAHPER (Canadian Association for Health Physical Education and Recreation. 1966), EUROFIT (Adam 1988), ALPHA (Ruiz et al. 2011)), with protocols recently developed for the preschool population (PREFIT (Ortega et al. 2015)). Our results are similar to field-test validation studies comparing vertical jump heights and sprint times against Wingate power outputs in older children, which found moderate to high correlations (Tharp et al. 1985, Hoffman et al. 2000, Bongers et al. 2015). In the current study, the $r=0.68$ correlation between LJ and PP is considerably higher than validation studies comparing LJ to 1RM leg extension or leg press reporting correlations from $\mathrm{r}=0.2-0.4$ in children age 6-12 (Milliken et al. 2008, Fernandez-Santos et al. 2015). This suggests that the LJ may be better able to predict lower body muscle power than tests of lower body muscle strength in young children. Although the field-tests in this study independently explained $40-45 \%$ of the variance in PP, adding 
participant's weight into the model significantly increased the predictive value of the tests; this is a common finding in the literature due to the high correlations between body mass and muscle power and strength measures, which is suggested to be due to increases in fat-free mass (Tharp et al. 1985, Fernandez-Santos et al. 2015). This is further supported by a recent study by Fry and colleagues (2015) who discovered that age related differences in leg strength and power in children from age 3 to 7 were minimal once body mass was adjusted for, this highlights the importance of considering body mass when interpreting muscular performance in young children.

The average peak power output of $94 \mathrm{~W}$ in the current sample is consistent with findings from Fry and colleagues (2015) who measured the highest mean power achieved during a maximal velocity leg press in a similar same age group (age 3-7). Previous research has also shown that laboratory-assessed peak and mean power using the aforementioned modified 10s Wingate protocol exhibits strong tracking, or stability of relative rankings over time, in preschool children (Gabel et al. 2011, Caldwell et al. 2016). Utilizing valid and reliable field-based tests in this age group will allow short-term muscle power to be tracked in large cohorts and ultimately help to determine its relevance to health-related outcomes later in life.

Due to the young age of the participants, a few children under the age of $4(n=15)$ had not yet achieved the level of coordination required to pedal the ergometer above $25 \mathrm{rpm}$ and were therefore excluded from the analysis. However, as many field-measures of MF involve one's ability to move in a coordinated way (e.g., long jump, vertical jump, sprinting), it would be expected that their ability to perform on the field-test would be hindered as well. This is 
supported by our data as these children who had difficulty pedaling had significantly shorter LJ distance $(\mathrm{t}=2.2, \mathrm{p}<.05)$ and longer SRT $(\mathrm{t}=3.2, \mathrm{p}<.01)$ than the 3 year old children who were able to pedal $>25 \mathrm{rpm}$. Although we cannot tease out the effects of motor coordination in the relationship between LJ and SRT to PP, both impaired motor coordination and low MF have been shown to be risk factors for negative health outcomes such as excess weight gain as children age (Ortega et al. 2008, Lopes et al. 2012). Although, within typically developing children, body weight is relevant to the prediction of absolute PP, overweight children who are at risk for poor MF would actually perform worse on the LJ and/or shuttle run. This would lead to an overall prediction of relative peak power $(\mathrm{W} / \mathrm{kg})$ much lower than a normal weight child of the same age who performs better on the LJ and shuttle run. By using field-tests of MF that incorporate dynamic coordination, teachers may be able not only to identify children with poor MF, but also identify children who may have concurrent motor coordination difficulties. These tests are valuable because both the LJ and BOT-2 shuttle run involve movement patterns that are typical of preschool-aged children and can be used to provide a reasonable estimate of peak muscle power in young children.

\section{Limitations}

To our knowledge, this is the first study to validate field-measures of MF to a laboratory criterion standard of short-term muscle power in preschool-aged children. Although the children were always given verbal encouragement and the tests were quick to complete and easy to comprehend, we recognize that due to the young age of the sample, participant motivation and attention may have impacted the children's performance. There may be small, random errors related to manual timing of the BOT-2 shuttle run and visual measurement of the LJ distance, 
however overall these should not impact the results as studies investigating the reliability of similar protocols have found no evidence of systematic bias or heteroscedasticity (Artero et al. 2011).

\section{Future directions}

The results from this study confirm that field-based assessments can be used to estimate peak muscle power in preschoolers. We recognize that there is limited evidence linking short-term muscle power in the early years to long-term health outcomes. Therefore, future research investigating the relationships between peak muscle power, as a surrogate of MF, and health outcomes, such as weight status or cardiovascular risk, in large samples of children is necessary before the predictive utility of these tests can be determined. As young children's activity patterns are characterized by short intermittent bursts of activity, their ability to create short-term power outputs may have important implications for their accumulation of physical activity throughout the day. Longitudinal studies examining these relationships starting at the preschool age are needed in order to ascertain the causal ordering and impact of low MF on physical activity and health from early childhood to adolescence. Future work examining the predictive validity of these field tests and the age cut-offs for poor peak muscle power are required before targeted interventions can be implemented. 


\section{Acknowledgements}

The Health Outcomes and Physical Activity in Preschoolers study was funded by the Canadian Institutes of Health Research (award \# 102560). BWT was supported by a CIHR New

Investigator Salary Award and an Early Researcher Award from the Ontario Ministry of Economic Development and Innovation.

\section{Conflict of Interest Disclaimer}

The authors report no conflicts of interest associated with this manuscript. 


\section{References}

Adam, C. 1988. Eurofit: Handbook for the eurofit tests of physical fitness. Italian National Olympic Committee, Central Direction for Sport's Technical Activities Documentation and Information Division.

Artero, E., Espana-Romero, V., Castro-Pinero, J., Ortega, F., Suni, J., Castillo-Garzon, M., and Ruiz, J. 2011. Reliability of field-based fitness tests in youth. Int. J. Sports Med. 32(3): 159.

Baquet, G., Stratton, G., Van Praagh, E., and Berthoin, S. 2007. Improving physical activity assessment in prepubertal children with high-frequency accelerometry monitoring: A methodological issue. Prev. Med. 44(2): 143-147. doi:10.1016/j.ypmed.2006.10.004.

Barnekow-Bergkvist, M., Hedberg, G., Pettersson, U., and Lorentzon, R. 2006. Relationships between physical activity and physical capacity in adolescent females and bone mass in adulthood. Scand. J. Med. Sci. Sports 16(6): 447-455. doi:10.1111/j.16000838.2005.00500.x.

Bar-Or, 0. 1987. The Wingate anaerobic test an update on methodology, reliability and validity. Sports Med. 4(6): 381-394.

Bénéfice, E., Fouéré, T., and Malina, R. 1999. Early nutritional history and motor performance of Senegalese children, 4-6 years of age. Ann. Hum. Biol. 26(5): 443455.

Bongers, B.C., Werkman, M.S., Blokland, D., Eijsermans, M.J., Van der Torre, P., Bartels, B., Verschuren, O., and Takken, T. 2015. Validity of the Pediatric Running-Based 
Anaerobic Sprint Test to Determine Anaerobic Performance in Healthy Children. Pediatr. Exerc. Sci. 27(2): 268-276. doi:10.1123/pes.2014-0078.

Bruininks, R.H., and Bruininks, B.D. 2005. Bruininks-Oseretsky Test of Motor Proficiency, Second Edition (BOT-2). Pearson Assessment, Minneapolis, MN.

Caldwell, H.A.T., Proudfoot, N.A., King-Dowling, S., Di Cristofaro, N.A., Cairney, J., and Timmons, B.W. 2016. Tracking of physical activity and fitness during the early years. Appl. Physiol. Nutr. Metab. doi:10.1139/apnm-2015-0338.

Canadian Association for Health Physical Education and Recreation. 1966. The CAHPER fitness-performance test manual for boys and girls 7 to 17 years of age. CAHPER, Ottawa.

Castro-Piñero, J., Artero, E.G., España-Romero, V., Ortega, F.B., Sjöström, M., Suni, J., and Ruiz, J.R. 2010a. Criterion-related validity of field-based fitness tests in youth: a systematic review. Br. J. Sports Med. 44(13): 934-943. doi:10.1136/bjsm.2009.058321.

Castro-Piñero, J., Ortega, F.B., Artero, E.G., Girela-Rejón, M.J., Mora, J., Sjöström, M., and Ruiz, J.R. 2010b. Assessing Muscular Strength in Youth: Usefulness of Standing Long Jump as a General Index of Muscular Fitness: J. Strength Cond. Res. 24(7): 1810-1817. doi:10.1519/JSC.0b013e3181ddb03d.

Fernandez-Santos, J.R., Ruiz, J.R., Cohen, D.D., Gonzalez-Montesinos, J.L., and Castro-Piñero, J. 2015. Reliability and Validity of Tests to Assess Lower-Body Muscular Power in Children: J. Strength Cond. Res. 29(8): 2277-2285. doi:10.1519/JSC.0000000000000864. 
Freedson, P.S., Cureton, K.J., and Heath, G.W. 2000. Status of Field-Based Fitness Testing in Children and Youth. Prev. Med. 31(2): S77-S85. doi:10.1006/pmed.2000.0650.

Fry, A.C., Irwin, C.C., Nicoll, J.X., and Ferebee, D.E. 2015. Muscular Strength and Power in 3to 7-Year-Old Children. Pediatr. Exerc. Sci. 27(3): 345-354. doi:10.1123/pes.20140152.

Hoffman, J.R., Epstein, S., Einbinder, M., and Weinstein, Y. 2000. A Comparison Between the Wingate Anaerobic Power Test to Both Vertical Jump and Line Drill Tests in Basketball Players. J. Strength Cond. Res. 14(3): 261-264.

Kemper, H.C.G., Twisk, J.W.R., van Mechelen, W., Post, G.B., Roos, J.C., and Lips, P. 2000. A fifteen-year longitudinal study in young adults on the relation of physical activity and fitness with the development of the bone mass: the Amsterdam Growth and Health Longitudinal Study. Bone 27(6): 847-853. doi:10.1016/S87563282(00)00397-5.

Kuczmarski, R.J., Ogden, C.L., Guo, S.S., Grummer-Strawn, L.M., Flegal, K.M., Mei, Z., Wei, R., Curtin, L.R., Roche, A.F., and Johnson, C.L. 2002. 2000 CDC Growth Charts for the United States: methods and development. Vital Health Stat. 11. (246): 1-190.

Lopes, Ví.P., Maia, J.A.R., Rodrigues, L.P., and Malina, R. 2012. Motor coordination, physical activity and fitness as predictors of longitudinal change in adiposity during childhood. Eur. J. Sport Sci. 12(4): 384-391. doi:10.1080/17461391.2011.566368. Milliken, L.A., Faigenbaum, A.D., Loud, R.L., and Westcott, W.L. 2008. Correlates of Upper and Lower Body Muscular Strength in Children: J. Strength Cond. Res. 22(4): 13391346. doi:10.1519/JSC.0b013e31817393b1. 
Nguyen, T., Obeid, J., and Timmons, B.W. 2011. Reliability of fitness measures in 3- to 5year-old children. Pediatr. Exerc. Sci. 23(2): 250-260.

Oja, L., and Jürimäe, T. 1997. Assessment of motor ability of 4 - and 5 - year - old children. Am. J. Hum. Biol. 9(5): 659-664.

Ortega, F.B., Cadenas-Sánchez, C., Sánchez-Delgado, G., Mora-González, J., Martínez-Téllez, B., Artero, E.G., Castro-Piñero, J., Labayen, I., Chillón, P., and Löf, M. 2015. Systematic review and proposal of a field-based physical fitness-test battery in preschool children: the PREFIT battery. Sports Med. 45(4): 533-555.

Ortega, F.B., Ruiz, J.R., Castillo, M.J., and Sjöström, M. 2008. Physical fitness in childhood and adolescence: a powerful marker of health. Int. J. Obes. 32(1): 1-11. doi:10.1038/sj.ijo.0803774.

Pate, R., Oria, M., and Pillsbury, L. 2012. Fitness measures and health outcomes in youth. National Academies Press.

Plowman, S.A. 2014. Top 10 Research Questions Related to Musculoskeletal Physical Fitness Testing in Children and Adolescents. Res. Q. Exerc. Sport 85(2): 174-187. doi:10.1080/02701367.2014.899857.

Ruiz, J.R., Castro-Piñero, J., España-Romero, V., Artero, E.G., Ortega, F.B., Cuenca, M.M., Jimenez-Pavón, D., Chillón, P., Girela-Rejón, M.J., Mora, J., Gutiérrez, Á., Suni, J., Sjöström, M., and Castillo, M.J. 2011. Field-based fitness assessment in young people: the ALPHA health-related fitness test battery for children and adolescents. Br. J. Sports Med. 45(6): 518-524. doi:10.1136/bjsm.2010.075341.

Safrit, M.J. 1990. The validity and reliability of fitness tests for children: a review. Pediatr. Exerc. Sci. 2(1): 9-28. 
Smith, J.J., Eather, N., Morgan, P.J., Plotnikoff, R.C., Faigenbaum, A.D., and Lubans, D.R. 2014. The Health Benefits of Muscular Fitness for Children and Adolescents: A Systematic Review and Meta-Analysis. Sports Med. 44(9): 1209-1223. doi:10.1007/s40279014-0196-4.

Tharp, G.D., Newhouse, R.K., Uffelman, L., Thorland, W.G., and Johnson, G.O. 1985. Comparison of Sprint and Run Times with Performance on the Wingate Anaerobic Test. Res. Q. Exerc. Sport 56(1): 73-76. doi:10.1080/02701367.1985.10608434.

Timmons, B.W., Proudfoot, N.A., MacDonald, M.J., Bray, S.R., and Cairney, J. 2012. The health outcomes and physical activity in preschoolers (HOPP) study: rationale and design. BMC Public Health 12: 284. doi:10.1186/1471-2458-12-284.

Van Praagh, E., and Doré, E. 2002. Short-term muscle power during growth and maturation. Sports Med. 32(11): 701-728. 
Table 1: Participant characteristics

\begin{tabular}{|c|c|c|}
\hline Variable & Mean \pm SD & Median (Range) \\
\hline Age (years) & $4.6 \pm 0.9$ & $4.7(3.0-6.0)$ \\
\hline Height (cm) & $107.1 \pm 7.5$ & $107.5(89.9-126.1)$ \\
\hline Weight (kg) & $18.1 \pm 3.1$ & $17.7(12.1-34.2)$ \\
\hline BMI (\%ile) ${ }^{*}$ & $52.7 \pm 28.4$ & $55.6(0.0-99.6)$ \\
\hline \multicolumn{3}{|c|}{ Fitness Measures } \\
\hline LJ (in) & $28.9 \pm 10.0$ & $31.0(3.0-54.0)$ \\
\hline SRT (s) & $12.0 \pm 2.0$ & $11.6(8.3-20.1)$ \\
\hline PP (W) & $93.9 \pm 37.6$ & $94.1(17.1-244.3)$ \\
\hline
\end{tabular}

*Based on the Centre for Disease Control data (Kuczmarski et al. 2002) BMI, body mass index; LJ, long jump; SRT, shuttle run time, PP, peak power 
Table 2. Regression models predicting PP (W) from age, sex, weight and field assessments in preschool children.

\begin{tabular}{|l|l|l|l|l|}
\hline & Model 1 & Model 2 & Model 3 & Model 4 \\
\hline & & & & \\
\hline Intercept & -111.01 & -4.87 & -97.68 & $-19.12^{* *}$ \\
\hline Age (years) & $0.32(1.34)^{* *}$ & $0.16(1.51)^{* *}$ & $0.20(1.52)^{* *}$ & $0.13(1.54)$ \\
\hline Sex & $0.06(1.83)^{*}$ & $0.05(1.68)^{*}$ & $0.05(1.73)^{*}$ & $0.05(1.65)^{*}$ \\
\hline Weight (kg) & $0.65(0.35)^{* *}$ & $0.61(0.32)^{* *}$ & $0.61(0.34)^{* *}$ & $0.58(0.32)^{* *}$ \\
\hline LJ (in) & & $0.23(0.12)^{* *}$ & $0.13(0.13)^{* *}$ \\
\hline SRT (s) & $-0.28(0.59)^{* *}$ & & $-0.23(0.66)^{* *}$ \\
\hline \multicolumn{5}{|l|}{} \\
\hline Adjusted R ${ }^{2}$ & $0.77^{* *}$ & $0.81^{* *}$ & $0.79^{* *}$ & $0.82^{* *}$ \\
\hline
\end{tabular}

Results presented as $\beta(\mathrm{SE}){ }^{*}=\mathrm{p}<.05, * *=\mathrm{p}<.01$; SRT, shuttle run time; LJ, long jump. Model 1 (age, sex and weight), $n=393$; model 2 (SRT), $n=380$; model 3 (LJ), $n=376$; model 4 (SRT and $\mathrm{LJ}), \mathrm{n}=366$ 